\title{
Assessment of thyroid dysfunction in the type 2 diabetic patients
}

\author{
P. Singh $\cdot$ S. Khan $\cdot$ R. K. Mittal
}

Received: 4 June 2013 / Accepted: 18 April 2014 / Published online: 8 May 2014

(C) Research Society for Study of Diabetes in India 2014

\section{Dear editor}

Thyroid dysfunction is found in Diabetes Mellitus (DM). Thyroid disorders are common and are second only to diabetes as the condition to affect the endocrine system. As a result it is common for an individual to be affected by both thyroid diseases and diabetes [1]. It is necessary to recognize and treat it when present in order to achieve stability of metabolic control. The objective of this study was to investigate the effect of diabetes mellitus on thyroid hormone levels and other biochemical variables. To determine the incidence of abnormal thyroid hormone levels in diabetics in Nepalgunj medical college and Hospital, Nepal, blood samples from 100 diabetic subjects and 100 non-diabetic controls were taken between 1st February, 2012 and 31st January, 2013 for free triidothyronine (FT3), free thyroxine (FT4), thyroid stimulating hormone (TSH), fasting plasma glucose (FPG), serum cholesterol, serum triglycerides, high density lipoprotein(HDL), low density lipoprotein(LDL), very low density lipoprotein(VLDL), blood urea, serum creatinine, total protein, albumin, SGOT and SGPT. Our findings showed that FPG, serum cholesterol, serum triglycerides, serum VLDL, serum

Table 1 Serum thyroid hormone levels in non-diabetic and diabetic subjects

\begin{tabular}{llll}
\hline Thyroid hormones & Diabetic subjects & Non-diabetic controls & P value \\
\hline FT3 & $2.57 \pm 0.74$ & $3.01 \pm 0.99$ & $<0.0001$ \\
FT4 & $1.32 \pm 0.27$ & $1.43 \pm 0.46$ & $\approx 0.0405$ \\
TSH & $5.54 \pm 2.24$ & $2.89 \pm 1.31$ & $<0.0001$ \\
\hline
\end{tabular}

P. Singh $(\bowtie) \cdot$ R. K. Mittal

Department of Biochemistry, Nepalgunj Medical College, Chisapani,

Banke, Nepal

e-mail: priti186631@gmail.com

S. Khan

Department of Microbiology, Nepalgunj Medical College,

Chisapani, Nepal
Table 2 Prevalence of thyroid disorder according to gender

\begin{tabular}{llll}
\hline $\begin{array}{l}\text { Diabetic subjects } \\
(n=100)\end{array}$ & Hypothyroidism & Hyperthyroidism & Prevalence \\
\hline Male & $8(8 \%)$ & $2(2 \%)$ & $10 \%$ \\
Female & $16(16 \%)$ & $3(3 \%)$ & $19 \%$ \\
Total & $24 \%$ & $5 \%$ & $29 \%$ \\
\hline
\end{tabular}

creatinine, blood urea, SGOT and SGPT were significantly higher in diabetic subjects as compared to non-diabetic controls while serum HDL, total protein and albumin was significantly lower in diabetic as compared to non-diabetic controls. The level of FT3 $(P<0.0001)$ and FT4 $(P \approx 0.0405)$ were significantly lower while the level of TSH $(P<0.0001)$ was significantly higher in type 2 diabetics as compared to non-diabetics (Table 1). From the 100 diabetic subjects studied, $29 \%$ showed abnormal thyroid hormone levels (24\% hypothyroidism and $5 \%$ hyperthyroidism). The incidence of hypothyroidism was more in females $(16 \%)$ as compare to males $(8 \%)$ in type 2 diabetes (Table 2). Failure to recognize the presence of these abnormal thyroid hormone levels in diabetics may be a primary cause of poor management often encountered in some treated diabetics. This study has shown a high incidence of abnormal thyroid hormone levels among diabetic patients as compared to non diabetics.

In conclusion, our findings demonstrate that detection of abnormal thyroid hormone levels in addition to other biochemical variables in the early stage of diabetes will help patients improve their health and reduce their morbidity rate.

Conflict of interest None.

\section{References}

1. Feely J, Isles TE. Screening for thyroid dysfunction in diabetics. Br Med J. 1979;1(6179):1678. 